\title{
Tea with Crows
}

\section{Describe the process of how this was made.}

"Tea with Crows" is an interactive sculpture that consists of two design components: a stand-alone sculpture and a kinetic chandelier that hangs from the ceiling. It took six months to construct both of the pieces. Using Rhinoceros 3D software, I designed and printed assemblages of over 223 plastic and metal pieces, connected the delicate segments, and constructed the mechanical movement of the swan and crows, while embedding the sensors and servo motors.

\section{What for you is the most important/interesting thing about what you made? \\ “Tea with Crows" engages with people through theat- rical staging. The sculpture comes to life when someone approaches it, displaying its various characteristics when they perform the drinking of tea with cups, the focus}

of participant engagement. It's very important for "Tea with Crows" to be engaging, enabling the audience to explore ambiguous interactivity metaphorically, aesthetically, and functionally. This allows people to creatively find ways to interact with the swan, which forms the tea table, and the crow, which makes up the structure of the chandelier. Through the meaning-making process of interaction, the audience is encouraged to redefine the idea of art, design, and everyday objects.

\section{Did anything go wrong?}

Of course, I always face challenges in the act of creation while following my imagination as an artist. However, it is very important for me to make my pieces with an open mind, leaving room for adaptation. I relish scavenging for solutions and materials, exploding my initial conceptions, and

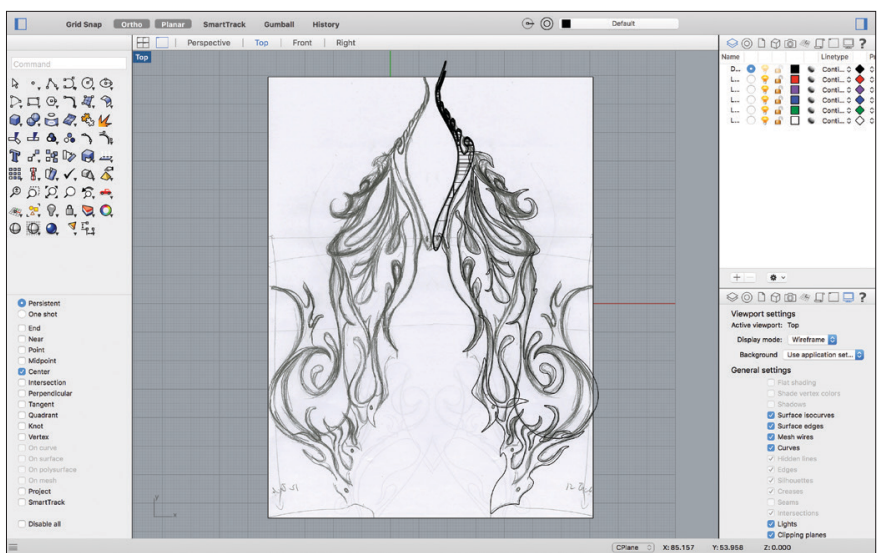

$\rightarrow$ Imported sketch in the 3D-modeling software. gaining knowledge through the process of making. I can say that this process sometimes produces unexpected richness in the "act of making." Originally, I pictured two layers of interaction for the chandelier, but the outer parts of the sculpture were simply too heavy to be held by servo motors. (The penguins were too heavy to fly!) Thus, the chandelier remained as one layer consisting of of three crows. But this configuration turned out to emphasize the crows' details very well, preventing them from being hidden by another layer.

\section{What was the biggest sur-} prise in making this?

I did not really intend it this way, but at the end of my working process, I realized that the bottom sculpture and the top sculpture have two very distinctive characters and evoke different feelings in the audience. What I have been hearing from people interacting with the piece is that the white swan motion is very elegant and graceful but the crows are creepy and menacing. I love seeing these dual elements, which were externalized from my ideas and personality.

\section{Was there anything new for} you in the making, process, materials, or something else that you can tell us about? Because of my experience in a traditional studio art context, my creative process inevitably illustrates the "act of studio practice" that is fundamental to me as an artist. There was also an intense post-production process involved in hiding the mechanical elements and concealing the digitally 3D-fabricated elements. For example, even though "Tea with Crows" was fabricated mainly with 3D-printed objects, completing it required several episodes of

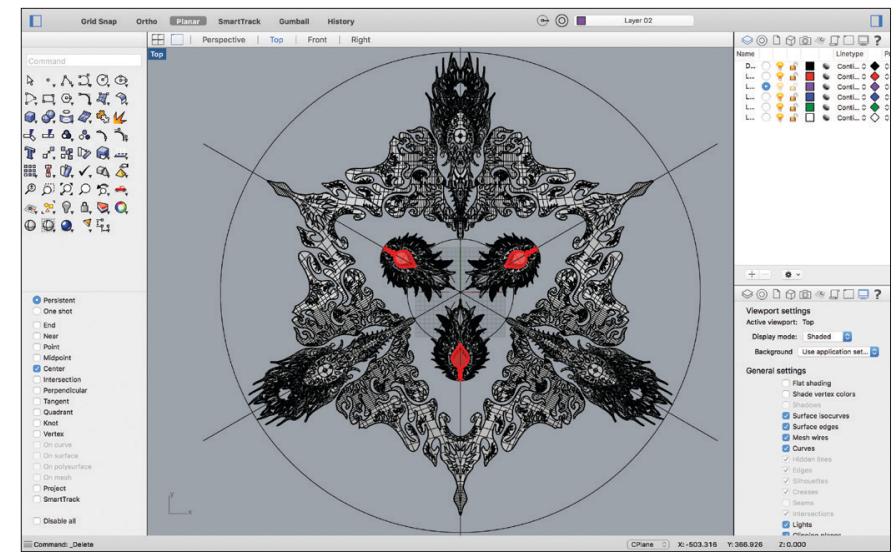

$\rightarrow$ 3D-modeling process for the Swan (tea table). 
paint brushing to achieve a more textured and organic surface. Otherwise, the $3 \mathrm{D}$-printed materials would have retained a digitally manufactured polished feel, which was unsuitable for my idea. Fabricating the pieces also required patience and a love of assemblage - so much a part of studio practice. At the same time, I was pursuing the sophisticated structures of the kinetic motions. The delicate mechanical segments were assembled by a hand-crafted process using metal wires, thread, epoxy glue, hinges, bolts, and nuts.

(1) Young Suk Lee, Indiana University, South Bend, $\rightarrow$ artdesignysdgmail.com

() http://www.youngsuklee.com/ TeawithCrows.html

(-) https://vimeo.com/216851928

DOI: $10.1145 / 3125397$ COPYRIGHT HELD BY AUTHOR
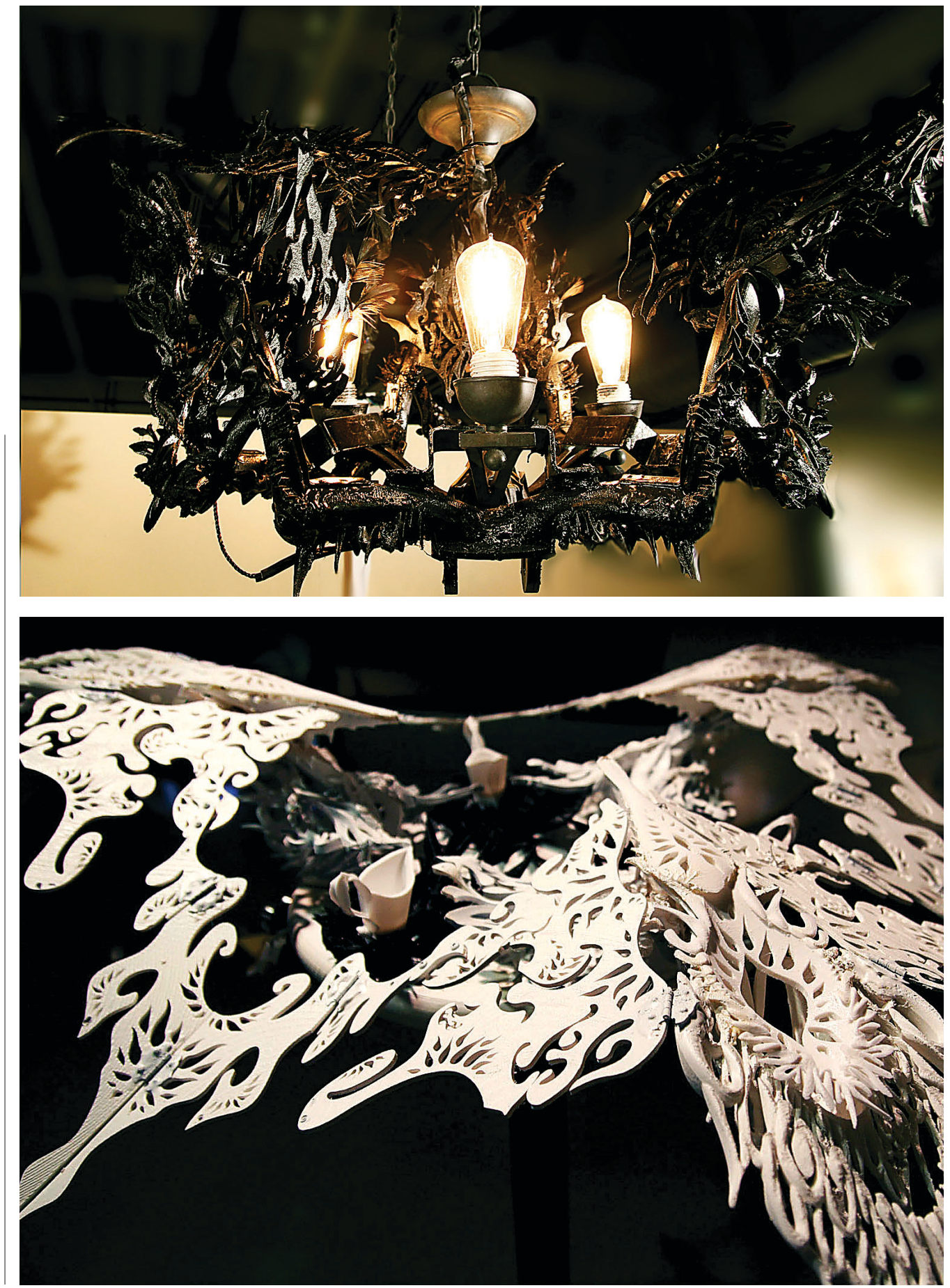

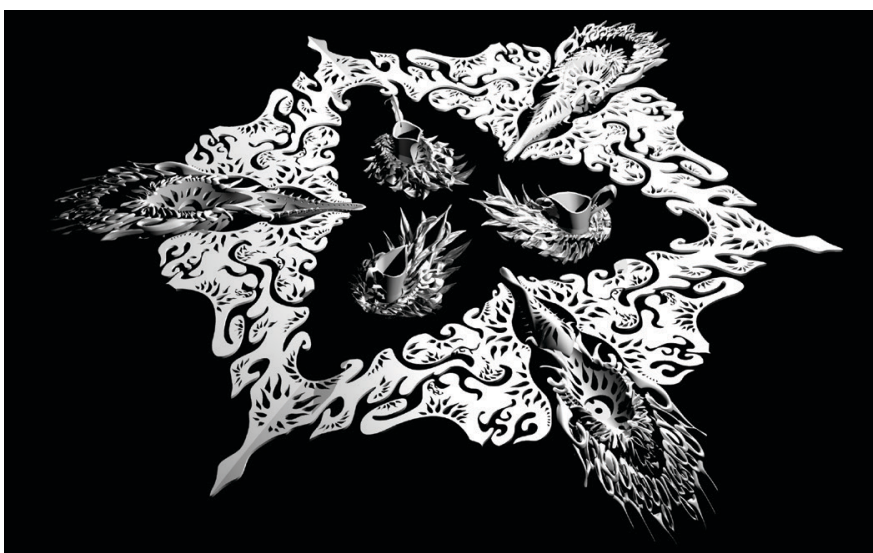

$\rightarrow$ 3D-rendered image of the Swan.

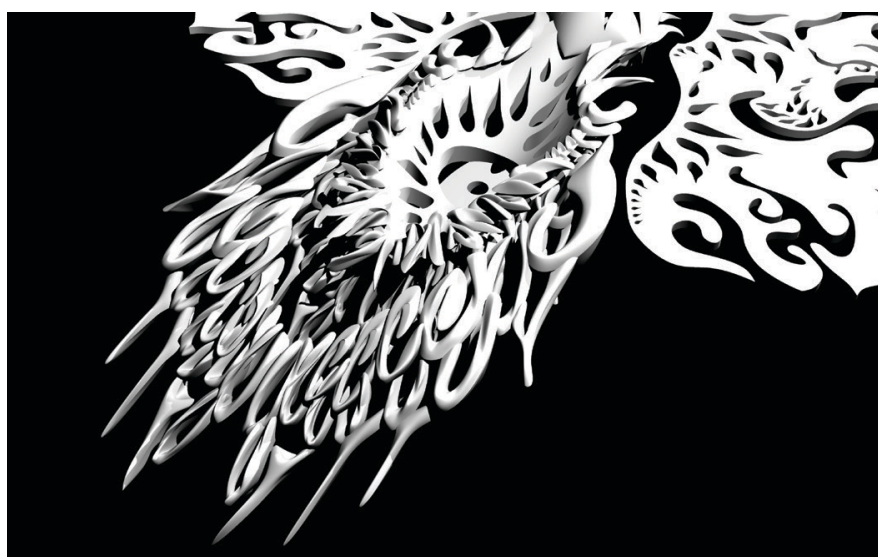

$\rightarrow$ Close-up shots of the 3D-rendered Swan. 